OPEN ACCESS

Edited by:

Jean-Paul Deslypere,

Proclin Therapeutic Research

Pvt. Ltd., Singapore

Reviewed by:

Stefano Giovagnoli,

University of Perugia, Italy

Gerfried Karl Hans Nell,

NPC Nell Pharma Connect Ltd.,

Austria

*Correspondence:

Jian Wang

jianwang08@163.com;

jianwang0815@sina.com

tThese authors have contributed equally to this work.

Specialty section

This article was submitted to

Pharmaceutical Medicine

and Outcomes Research,

a section of the journal

Frontiers in Pharmacology

Received: 10 March 2017

Accepted: 07 July 2017

Published: 29 August 2017

Citation:

Ma X, Yang YX, Chen N, Xie Q,

Wang T, He $X$ and Wang $J(2017)$

Meta-Analysis for Clinical Evaluation

of Xingnaojing Injection

for the Treatment of Cerebral

Infarction. Front. Pharmacol. 8:485.

doi: 10.3389/fphar.2017.00485

\section{Meta-Analysis for Clinical Evaluation of Xingnaojing Injection for the Treatment of Cerebral Infarction}

\author{
Xiao Ma ${ }^{1 t}$, Yu X. Yang ${ }^{1 \dagger}$, Nian Chen ${ }^{1}$, Qian Xie ${ }^{1}$, Tao Wang ${ }^{1}$, Xuan $\mathrm{He}^{2}$ and Jian Wang ${ }^{1 *}$ \\ ' College of Pharmacy, Chengdu University of Traditional Chinese Medicine, Chengdu, China, ${ }^{2}$ Department of Pharmacy, \\ Xindu District Shibantan Public Hospital, Chengdu, China
}

Objective: Xingnaojing injection (XNJ) is derived from An-Gong-Niu-Huang pill, a wellknown traditional Chinese patent medicine, which is widely used for stroke. To evaluate the therapeutic effect of XNJ on cerebral infarction, an extensive meta-analysis was used.

Methods: Six major electronic databases including the Chinese Biomedical Database (CBM), Wanfang, the VIP medicine information system (VMIS) and the China National Knowledge Infrastructure (CNKI), PubMed, Embase, and the Cochrane Library were examined to retrieve randomized controlled trials designed to evaluate the clinical efficacy of XNJ in treating Cl before November 26, 2016.

Results: There were 53 randomized controlled trials with 4915 participants in this study. The results reflected that compared with the conventional therapy (CT) alone, XNJ could significantly improve the overall response rate $(O R=3.56,95 \% \mathrm{Cl}[2.94$, 4.32], $P<0.00001$ ), and clinical symptom (including increasing activities of daily living (ADL, $M D=10.23,95 \% \mathrm{Cl}[9.47,10.99], P<0.00001$ ), and reduce infarction size $(M D=-1.83,95 \% \mathrm{Cl}[-2.49,-1.16], P<0.00001))$. However, there was no significant difference between the XNJ treatment and conventional therapy in Glasgow Coma Scale (GCS, $P=0.32$ ). Neurological deficit score demonstrated that XNJ could significantly reduce the score in two different evaluation criterions as National Institutes of Health Stroke Scale (NIHSS, MD $=-3.44,95 \% \mathrm{Cl}[-4.52,-2.36], P<0.00001)$, and the Chinese Stroke Scale (CSS, MD $=-5.72,95 \% \mathrm{Cl}[-6.94,-4.50], P<0.00001$ ). Additionally, serum MMPs, including MMP-2 and MMP-9 were significantly reduced by $\mathrm{XNJ}$ treatment compared with conventional therapy $(M D=-11.24,95 \% \mathrm{Cl}$ [-20.83, -1.65], $P=0.02 ; M D=-25.08,95 \% \mathrm{Cl}[-35.49,-14.67], P<0.00001$, respectively). Moreover, XNJ was able to improve hemorrheology in reducing whole blood viscosity, plasma viscosity, and hematocrit $(M D=-1.44,95 \% \mathrm{Cl}[-2.18$, $0.70], P=0.001 ; M D=-0.22,95 \% \mathrm{Cl}[-0.37,-0.07], P=0.003 ; M D=-3.63$, $95 \% \mathrm{Cl}[-6.23,-1.03], P=0.006$, respectively). The therapeutic efficacy of XNJ was found associated with improving hemodynamics (increasing peak-flow rate, and average velocity) $(M D=12.66,95 \% \mathrm{Cl}[10.50,14.81], P<0.00001 ; M D=9.90$, 
95\% $\mathrm{Cl}[8.63,11.17], P<0.00001)$. XNJ was also related to reducing cholesterol and triglyceride $(M D=-1.06,95 \% \mathrm{Cl}[-1.21,-0.92], P<0.00001 ; M D=-1.05,95 \% \mathrm{Cl}$ $[-1.12,-0.97], P<0.00001)$.

Conclusion: Despite the sample size and the poor quality of the included studies of this review, the results of the research showed that XNJ might be a beneficial therapeutic method for the treatment of cerebral infarction.

Keywords: Xingnaojing injection, cerebral infarction, meta-analysis, systematic review, clinical evaluation

\section{INTRODUCTION}

Stroke is the one of the most common diseases worldwide, with a high disability, mortality and recurrence rate, and usually leads to serious damage of central nervous system (Johnston et al., 2009). There are three types of stroke, including ischemia stroke, cerebral hemorrhage, and cerebral thrombosis. Ischemia stroke, also been known as cerebral infarction, is the most commonly seen in stroke, with the highest morbidity about $70 \%$ (Luo, 2010). Although the main pathology clearly defined, namely that brain tissue hypoxic ischemia is caused by atherosclerosis of cerebral arteries, alone or with superimposed thrombosis, hypertension, diabetes mellitus, and cardiovascular disease (Zhou, 2010; Hata et al., 2011), the corresponding precautionary measures are still limited in preventing the incidence rate.

Currently, the conventional therapy (CT), including thrombolysis, restoring blood supply to ischemic area, controlling cerebral edema, cerebral protection agents, preventing and treating complications, controlling hypertension, reducing blood viscosity, and so on, is the main clinical therapy for cerebral infarction (Deng et al., 2011). Recently, the theory that complementary medicine can substantial improve the disease has been put forward (Sze et al., 2005).

Xingnaojing injection (XNJ) is composed of musk, synthetic borneol, Curcuma aromatica Salisb, and Gardenia jasminoides J.Ellis derived from a classic traditional Chinese emergency prescription named An-Gong-Niu-Huang pill. It is widely used to treat nervous system disorders in China (Guo et al., 2014). Numerous studies indicating that the main active ingredients are muscone, borneol, camphor, curcumin, and curzerenone, and demonstrated borneol could be as a quality control substance, and it should be no less than $0.7 \mathrm{mg}$ per $\mathrm{ml} \mathrm{XNJ} \mathrm{injection.} \mathrm{According} \mathrm{to} \mathrm{the} \mathrm{above,} \mathrm{the} \mathrm{clinical}$ widely used of $\mathrm{XNJ}$ are the quality assurance. In recent years, there was an increase of clinical and pharmacological research indicating that $\mathrm{XNJ}$ can ameliorate brain function and promote the recovery of consciousness. The mechanisms might include scavenging free radicals, improving cerebral edema and hypoxia, and increasing the metabolic rate and activity of brain cells (He et al., 2006, 2007; Guo, 2013; Ma et al., 2014). However, there is no comprehensive and systematic evidence to confirm its clinical efficacy. Therefore, a comprehensive meta-analysis was performed in order to systematically evaluate the effectiveness of XNJ combined with CT for the treatment of cerebral infarction compared with the CT alone.

\section{MATERIALS AND METHODS}

\section{The Literature Search Strategy}

All RCTs concerning the effectiveness and safety of $\mathrm{XNJ}$ in treating cerebral infarction were retrieved from six databases, including PubMed, EMBASE, Cochrane Library, Wanfang database, VIP medicine information system, and China National Knowledge Infrastructure Database (CNKI) from inception to November 26, 2016, in terms of the keywords of "Xingnaojing injection" [Title/Abstract] AND "ischemia stroke" [Title/Abstract] OR "cerebral infarction" [Title/Abstract]. The searched results were downloaded for the further screening.

\section{Inclusion and Excluded Criteria}

The literatures screening was performed by two investigators (Yuxue Yang and Tao Wang) independently. The title, abstract, and full-text were browsed in sequence to evaluate whether the study should be included according to the inclusion and exclusion criteria. The screening criteria were predesigned before screening, and the discussion was organized to evaluate the appropriateness of the studies if any divergences were observed.

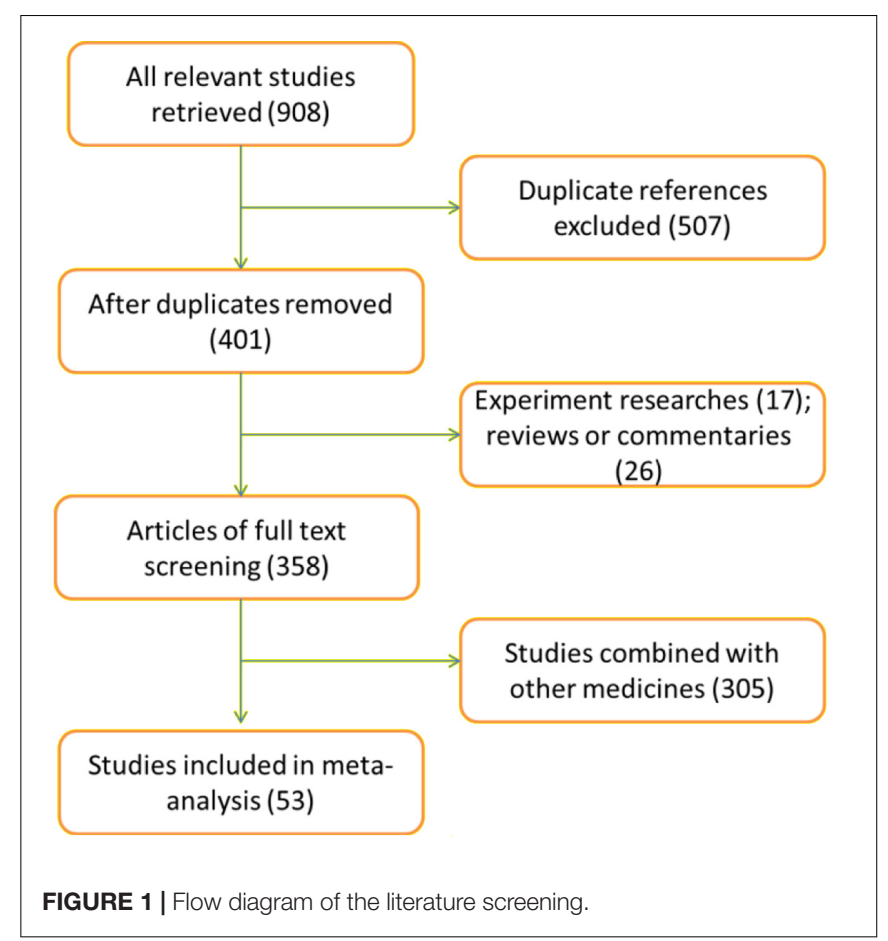


TABLE 1 | Characteristics and outcome measures of included studies.

\begin{tabular}{|c|c|c|c|c|c|}
\hline Included research (year) & $\mathrm{E} / \mathrm{C}$ & Intervening measure (E/C) & Dosage of $\mathrm{XNJ}$ & Duration & Outcome measures \\
\hline Wu et al., 1999 & $20 / 20$ & XNJ+ CT vs. CT & 40 mL/day & 15 days & Overall response rate \\
\hline Chen, 2006 & $39 / 30$ & $\mathrm{XNJ}+\mathrm{CT}$ vs. CT & $20 \mathrm{~mL} /$ day & 20 days & Overall response rate, Hemorheology, Blood lipid \\
\hline Zhang et al., 2006 & $32 / 28$ & $\mathrm{XNJ}+\mathrm{CT}$ vs. CT & $30 \mathrm{~mL} /$ day & 15 days & Neurological deficit score \\
\hline Li et al., 2008 & $27 / 27$ & $\mathrm{XNJ}+\mathrm{CT}$ vs. CT & $20 \mathrm{~mL} /$ day & 2 weeks & GCS \\
\hline Su, 2008 & $39 / 30$ & $\mathrm{XNJ}+\mathrm{CT}$ vs. CT & $20 \mathrm{~mL} /$ day & 20 days & Overall response rate \\
\hline Wu, 2008 & $40 / 40$ & $\mathrm{XNJ}+\mathrm{CT}$ vs. CT & $20 \mathrm{~mL} /$ day & 2 weeks & Overall response rate \\
\hline Chen and Wang, 2009 & $73 / 84$ & $\mathrm{XNJ}+\mathrm{CT}$ vs. CT & $30 \mathrm{~mL} /$ day & 2 weeks & Neurological deficit score \\
\hline Lu and Zhang, 2009 & $76 / 64$ & $\mathrm{XNJ}+\mathrm{CT}$ vs. CT & $40 \mathrm{~mL} /$ day & 15 days & Neurological deficit score \\
\hline Qiu, 2009 & $30 / 30$ & XNJ+ CT vs. CT & $20 \mathrm{~mL} /$ day & 3 weeks & Hemorheology, Overall response rate, Blood lipid \\
\hline Wu, 2009 & $54 / 52$ & $\mathrm{XNJ}+\mathrm{CT}$ vs. CT & $20 \mathrm{~mL} /$ day & 2 weeks & Overall response rate, neurological deficit score \\
\hline Jiang, 2010 & $30 / 28$ & $\mathrm{XNJ}+\mathrm{CT}$ vs. CT & $20 \mathrm{~mL} /$ day & 15 days & Overall response rate \\
\hline Lian and Dong, 2010 & $50 / 50$ & $\mathrm{XNJ}+\mathrm{CT}$ vs. CT & 30 mL/day & 2 weeks & Overall response rate, neurological deficit score \\
\hline Chen, 2011 & $30 / 30$ & $\mathrm{XNJ}+\mathrm{CT}$ vs. CT & 20 mL/day & 2 weeks & Overall response rate, neurological deficit score \\
\hline Han et al., 2011 & $43 / 43$ & $\mathrm{XNJ}+\mathrm{CT}$ vs. CT & $20 \mathrm{~mL} /$ day & 20-28 days & Neurological deficit score, Overall response rate \\
\hline Li and Hou, 2011 & $30 / 30$ & $\mathrm{XNJ}+\mathrm{CT}$ vs. CT & $20 \mathrm{~mL} /$ day & 2 weeks & Neurological deficit score, Overall response rate \\
\hline Qin et al., 2011 & $40 / 40$ & $\mathrm{XNJ}+\mathrm{CT}$ vs. CT & 20-30 mL/day & 2 weeks & Overall response rate, neurological deficit score \\
\hline Tong and Zhu, 2011 & $70 / 66$ & $\mathrm{XNJ}+\mathrm{CT}$ vs. CT & $30 \mathrm{~mL} /$ day & 2 weeks & Overall response rate, neurological deficit score \\
\hline Wang, 2011 & $30 / 30$ & $\mathrm{XNJ}+\mathrm{CT}$ vs. CT & $20 \mathrm{~mL} /$ day & 3 weeks & $\begin{array}{l}\text { Hemorheology, Blood lipid, neurological deficit } \\
\text { score }\end{array}$ \\
\hline Wang, 2011 & $40 / 40$ & $\mathrm{XNJ}+\mathrm{CT}$ vs. CT & $20 \mathrm{~mL} /$ day & 2 weeks & Overall response rate, neurological deficit score \\
\hline Yang, 2011 & $62 / 62$ & $\mathrm{XNJ}+\mathrm{CT}$ vs. CT & $20 \mathrm{~mL} /$ day & 15 days & Overall response rate \\
\hline Zang et al., 2011 & $49 / 49$ & XNJ+ CT vs. CT & $20 \mathrm{~mL} /$ day & 2 weeks & Overall response rate \\
\hline Li et al., 2012 & $43 / 43$ & XNJ+ CT vs. CT & $20 \mathrm{~mL} /$ day & 2 weeks & Neurological deficit score, Overall response rate \\
\hline Li et al., 2012 & $30 / 31$ & $\mathrm{XNJ}+\mathrm{CT}$ vs. CT & $20 \mathrm{~mL} /$ day & 2 weeks & $\begin{array}{l}\text { Neurological deficit score, Overall response rate, } \\
\text { Blood lipid }\end{array}$ \\
\hline Tong and Zhu, 2012 & $64 / 66$ & $\mathrm{XNJ}+\mathrm{CT}$ vs. CT & $30 \mathrm{~mL} /$ day & 2 weeks & Overall response rate, neurological deficit score \\
\hline Wang, 2012 & $100 / 100$ & $\mathrm{XNJ}+\mathrm{CT}$ vs. CT & $20 \mathrm{~mL} /$ day & 30 days & NIHSS, Overall response rate \\
\hline Dong and Fu, 2013 & $35 / 33$ & $\mathrm{XNJ}+\mathrm{CT}$ vs. CT & $20 \mathrm{~mL} /$ day & 2 weeks & Neurological deficit score \\
\hline Lou, 2013 & $75 / 75$ & XNJ+ CT vs. CT & $40 \mathrm{~mL} /$ day & 2 weeks & Overall response rate, neurological deficit score \\
\hline Qian and Jia, 2013 & $40 / 40$ & XNJ+ CT vs. CT & $20 \mathrm{~mL} /$ day & 2 weeks & Overall response rate, neurological deficit score \\
\hline Zeng, 2013 & $49 / 49$ & $\mathrm{XNJ}+\mathrm{CT}$ vs. CT & $20 \mathrm{~mL} /$ day & 2 weeks & Overall response rate \\
\hline Jia et al., 2014 & $96 / 96$ & $\mathrm{XNJ}+\mathrm{CT}$ vs. CT & $20 \mathrm{~mL} /$ day & 2 weeks & Infarction size, neurological deficit score \\
\hline Luo, 2014 & $60 / 60$ & $\mathrm{XNJ}+\mathrm{CT}$ vs. CT & $20 \mathrm{~mL} /$ day & 2 weeks & $\begin{array}{l}\text { Neurological deficit score, Overall response rate, } \\
\text { ADL, Hemorheology }\end{array}$ \\
\hline LV, 2014 & $62 / 62$ & $\mathrm{XNJ}+\mathrm{CT}$ vs. CT & 20 mL/day & 15 days & Overall response rate, MMPs \\
\hline Shen et al., 2014 & $45 / 41$ & $\mathrm{XNJ}+\mathrm{CT}$ vs. CT & $20 \mathrm{~mL} /$ day & 2 weeks & Overall response rate, GCS \\
\hline Sun, 2014 & $38 / 38$ & $\mathrm{XNJ}+\mathrm{CT}$ vs. CT & $20 \mathrm{~mL} /$ day & 2 weeks & Overall response rate, MMPs \\
\hline Wang et al., 2014 & $30 / 30$ & $\mathrm{XNJ}+\mathrm{CT}$ vs. CT & $20-30 \mathrm{~mL} /$ day & 2 weeks & Overall response rate, neurological deficit score \\
\hline Wei and Shi, 2014 & $50 / 50$ & $\mathrm{XNJ}+\mathrm{CT}$ vs. CT & 40 mL/day & 1 week & NHISS \\
\hline Yin et al., 2014 & $52 / 52$ & $\mathrm{XNJ}+\mathrm{CT}$ vs. CT & $20 \mathrm{~mL} /$ day & 2 weeks & Overall response rate, MMP-9 \\
\hline Yin and Wei, 2014 & $20 / 20$ & XNJ+ CT vs. CT & $20 \mathrm{~mL} /$ day & 2 weeks & Overall response rate, NHISS \\
\hline Zhang, 2014 & $49 / 49$ & XNJ+ CT vs. CT & $10 \mathrm{~mL} /$ day & 30 days & Neurological deficit score \\
\hline An et al., 2015 & $64 / 64$ & $\mathrm{XNJ}+\mathrm{CT}$ vs. CT & $20 \mathrm{~mL} /$ day & 2 weeks & $\begin{array}{l}\text { Overall response rate, Infarction size, neurological } \\
\text { deficit score }\end{array}$ \\
\hline Liao et al., 2015 & $24 / 23$ & $\mathrm{XNJ}+\mathrm{CT}$ vs. CT & $20 \mathrm{~mL} /$ day & NR & Overall response rate \\
\hline Luo et al., 2015 & $50 / 50$ & $\mathrm{XNJ}+\mathrm{CT}$ vs. CT & $10-20 \mathrm{~mL} /$ day & 4 weeks & Overall response rate, Hemodynamics \\
\hline Sun et al., 2015 & $42 / 42$ & XNJ+ CT vs. CT & $20 \mathrm{~mL} /$ day & NR & MMP-9, Overall response rate \\
\hline Wang and Huang, 2015 & $60 / 60$ & $\mathrm{XNJ}+\mathrm{CT}$ vs. CT & $10-20 \mathrm{~mL} /$ day & 20 days & Neurological deficit score, ADR \\
\hline Zhao et al., 2015 & $35 / 35$ & $\mathrm{XNJ}+\mathrm{CT}$ vs. CT & $20 \mathrm{~mL} /$ day & 2 weeks & Overall response rate, neurological deficit score \\
\hline Cai and Zhao, 2016 & $58 / 58$ & $\mathrm{XNJ}+\mathrm{CT}$ vs. CT & $20 \mathrm{~mL} / \mathrm{d}$ & 2 weeks & MMPs, Overall response rate \\
\hline Lin, 2016 & $30 / 30$ & XNJ+ CT vs. CT & 20 mL/day & 10 days & Neurological deficit score \\
\hline Lu et al., 2016 & $35 / 35$ & XNJ+ CT vs. CT & 30 mL/day & 2 weeks & $\begin{array}{l}\text { Overall response rate, neurological deficit score, } \\
\text { Infarction size }\end{array}$ \\
\hline
\end{tabular}


TABLE 1 | Continued

\begin{tabular}{|c|c|c|c|c|c|}
\hline Included research (year) & E/C & Intervening measure (E/C) & Dosage of XNJ & Duration & Outcome measures \\
\hline Lu et al., 2016 & $35 / 35$ & $\mathrm{XNJ}+\mathrm{CT}$ vs. CT & $30 \mathrm{~mL} /$ day & 2 weeks & ADL \\
\hline Wang and Wang, 2016 & $72 / 72$ & $\mathrm{XNJ}+\mathrm{CT}$ vs. CT & $20 \mathrm{~mL} /$ day & 30 days & Hemodynamics, Overall response rate \\
\hline Wang and Wang, 2016 & $40 / 40$ & $\mathrm{XNJ}+\mathrm{CT}$ vs. CT & $20 \mathrm{~mL} /$ day & 2 weeks & Overall response rate \\
\hline Xiong et al., 2016 & $48 / 48$ & $\mathrm{XNJ}+\mathrm{CT}$ vs. CT & $20 \mathrm{~mL} /$ day & 2 weeks & Overall response rate \\
\hline Yang, 2016 & $40 / 40$ & $\mathrm{XNJ}+\mathrm{CT}$ vs. CT & $20 \mathrm{~mL} /$ day & 15 days & Overall response rate \\
\hline
\end{tabular}

E, experimental group; C, control group; NR, no report.

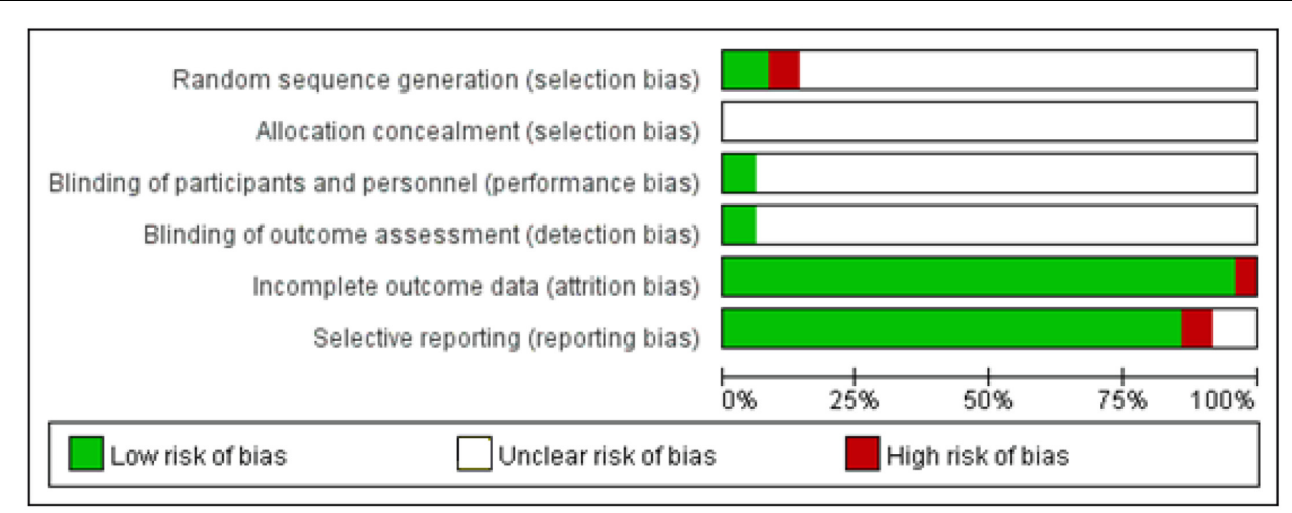

FIGURE 2 | Methodological quality assessment of the risk of bias for each included study.

\section{Inclusion Criteria}

The inclusion criteria were as follows: (1) Randomized controlled trials (RCTs); (2) Patients who were suffering from cerebral infarction according specific diagnosis criteria; (3) There were no other medicines in combination with the CT in the experimental group, except for Xingnaojing injection, compared with the CT as a control; (4) Treatment duration had to be at least 14 days; (5) One or more outcome measures, including the overall response rate, neurological deficit score, serum level of matrix metalloproteinase (MMPs), hemorheology, blood lipid, hemodynamic, clinical symptom improvement (including activities of daily living, ADL; Glasgow Coma Scale, GCS; and infarction size) a must be included in each study.

\section{Exclusion Criteria}

The study met with the following items would be excluded: (1) Duplicated articles, reviews, non-clinical studies, case observations; (2) Trials were not RCTs; (3) Combined with any other specific medicines in control group or experimental during the treatment.

\section{Data Extraction and Risk of Bias Assessment}

Two investigators (Yuxue Yang and Tao Wang) independently performed the data extraction and quality assessment of the included studies. The baseline information, including first author, publication year, the cases of experimental and control groups, respectively, interventions of experimental group, outcome measures were extracted to yield a conclusion table.
All the included RCTs were assessed for methodological quality using the Cochrane Handbook for Systematic Reviews of Interventions (Higgins et al., 2003). Five items, including random sequence generation, allocation concealment, blinding of participants and personnel, blinding of outcome assessment, incomplete outcome data and selective reporting were used for the methodological quality of each included studies. The quality of each item was assessed using the three levels of "low risk," "high risk," or "unclear risk."

\section{Data Analysis}

Cochrane Review Manager 5.3 (Cochrane Collaboration) was used for statistical analysis. Dichotomous variables, such as overall response rate, were presented as odds ratio $(O R)$, while the continuous variables, including the neurological deficit score, serum level of matrix metalloproteinase (MMPs), hemorheology, blood lipid, hemodynamic were presented as mean difference $(M D)$ with $95 \%$ confidence intervals (95\% CI). Additionally, the statistical heterogeneity was estimated using $P$-value and $I$-square $\left(I^{2}\right)$ statistic tests. Data with $P \geq 0.10$ and $I^{2} \leq 50 \%$ were defined as low heterogeneity, and a fixed-effects model would be used for the meta-analysis, whereas, a random-effects model was used for data with substantial heterogeneity $\left(P<0.10, I^{2}>50 \%\right)$. The publication bias was estimated by a funnel plot.

\section{RESULTS}

\section{Search Results}

Nine hundred and eight publications were retrieved according to the search strategy. After the title and abstract browsing, 507 


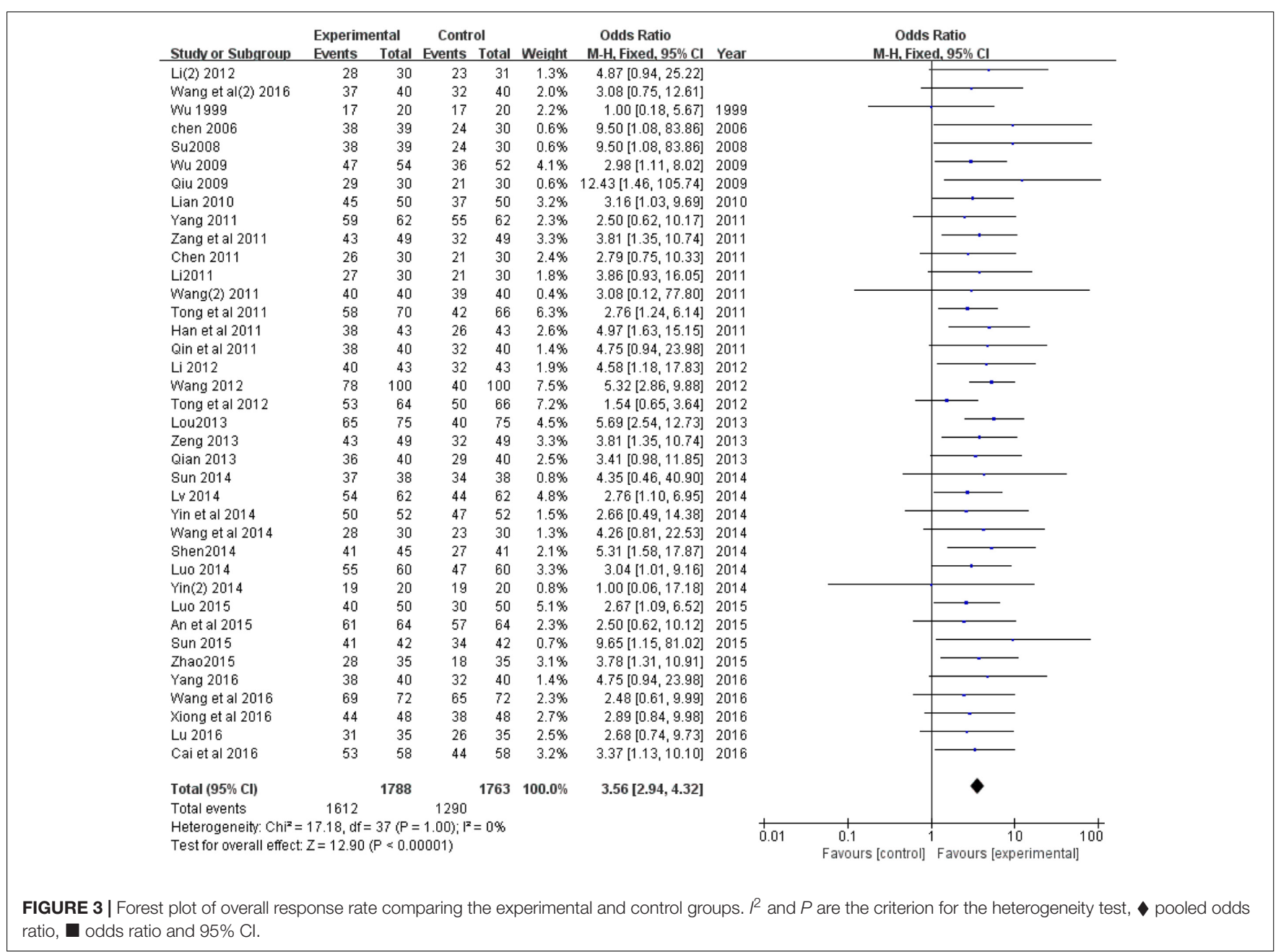

articles were removed for duplicated. 348 reviews were excluded for the following reasons: non-randomized controlled studies, animal studies, ambiguous outcome measures and overlapping data with another study, and combination with other medicines. Ultimately, 53 eligible articles with 4915 participants ( 2475 cases in the experimental group, 2440 cases in the control group) were included in the meta-analysis according to the inclusion and exclusion criteria after the full-text reading (Figure 1).

\section{Study Characteristics and Methodological Quality of Included Trials}

Among the 53 included trails, which were published between 1999 and 2016, all were RCTs with a comparison between a combination of XNJ and CT with CT treatment alone. The dosage of administered XNJ ranged from 10 to $40 \mathrm{~mL} /$ day via intravenous drip. There was no significant difference between the experimental and control groups in general information (Table 1).

The methodological quality of included studies was estimated according to the Cochrane risk of bias estimation. All of the included trails mentioned randomized allocation, whereas, only 5 of them mentioned the appropriate generation of the random allocation sequence (Qin et al., 2011; Dong and Fu, 2013; Liao et al., 2015; Lu and Liu, 2016; Lu et al., 2016). There was no trails mentioned allocation concealment. Three of the 53 studies stated double blind design (blinding of participants and blinding of outcome assessment) (Li et al., 2008; Chen and Wang, 2009; $\mathrm{Lv}, 2014)$. Two of 53 studies were published with a high risk of incomplete outcome (Han et al., 2011; Wang and Jiang, 2016), and the risk of selective reporting was high for the three trials (Lian and Dong, 2010; Chen, 2011; Wang, 2012) (Figure 2).

\section{Analysis and Subgroup Analysis for Outcome Measures \\ The Overall Response Rate}

Thirty-eight of the 53 trials (Wu et al., 1999; Chen, 2006, 2011; Su, 2008; Wu, 2008; Qiu, 2009; Lian and Dong, 2010; Han et al., 2011; Li and Hou, 2011; Qin et al., 2011; Tong and Zhu, 2011; Wang, 2011, 2012; Yang, 2011, 2016; Zang et al., 2011; Li and Su, 2012; Li et al., 2012; Tong and Zhu, 2012; Lou, 2013; Qian and Jia, 2013; Zeng, 2013; Luo, 2014; Lv, 2014; Shen et al., 2014; Sun, 2014; Wang et al., 2014; Yin and Wei, 2014; Yin et al., 2014; An et al., 2015; Luo et al., 2015; Sun et al., 2015; Zhao et al., 2015; Cai and Zhao, 2016; Lu et al., 


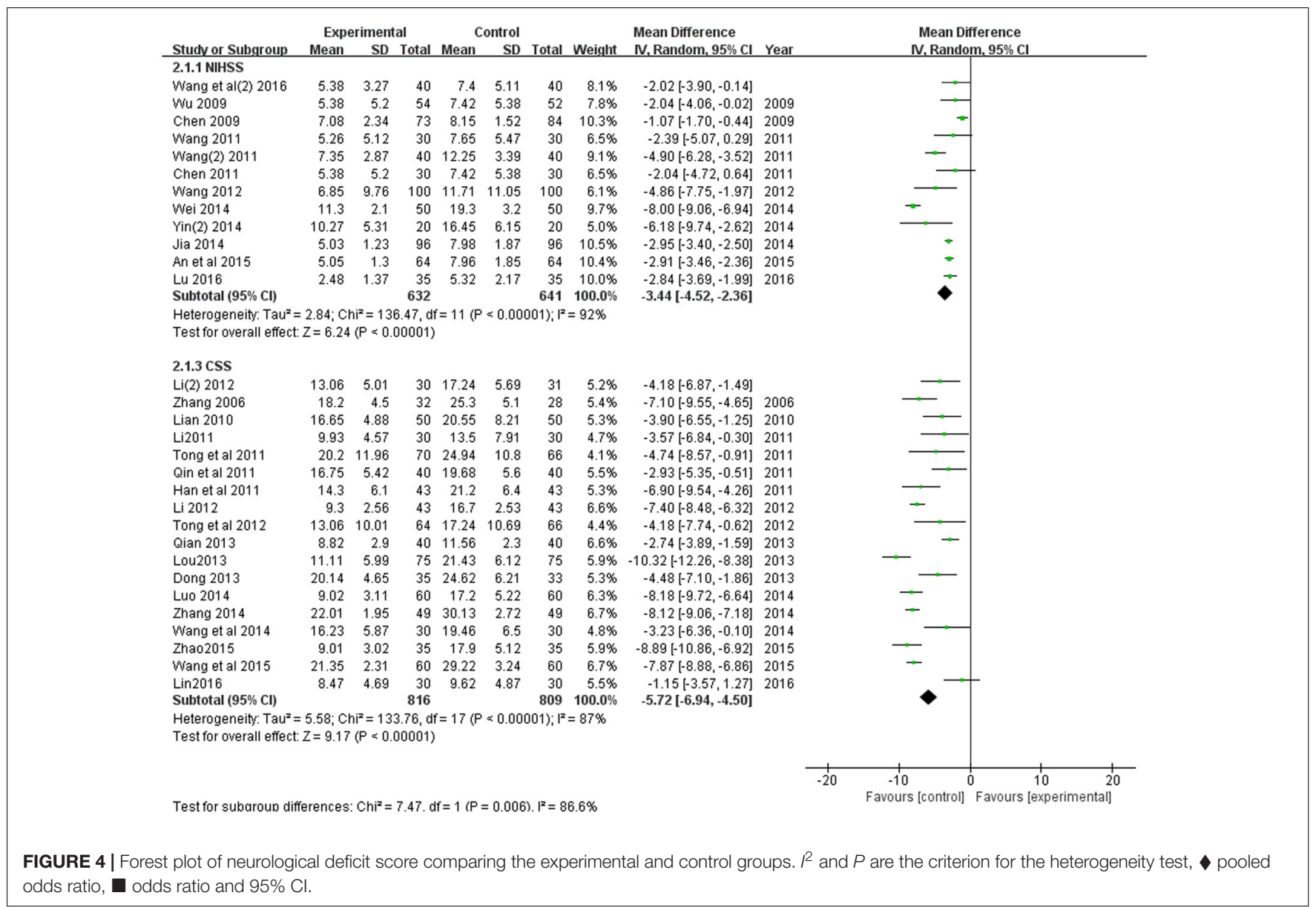

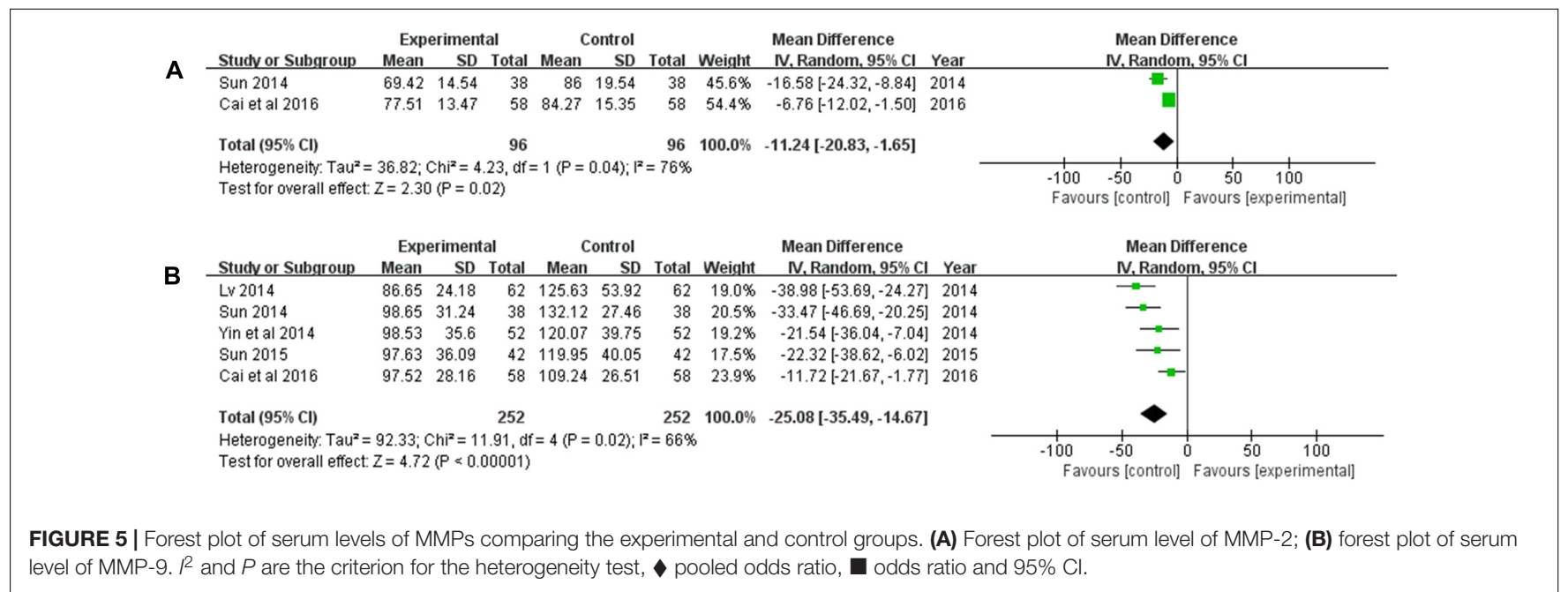

2016; Wang and Jiang, 2016; Wang and Wang, 2016; Xiong et al., 2016) compared the overall response rate between XNJ combined with CT treatment and single CT treatment. There was no heterogeneity $\left(P=1.0, I^{2}=0 \%\right)$, and a fixed-effect model was used to carry out the meta-analysis. An $O R$ with 95\% CI was used to present the comparison of overall response rate between the experimental and control groups $(O R=3.56$,
95\% CI [2.94, 4.32], $P<0.00001)$. It revealed that XNJ could significantly increase the treatment efficacy of CT for cerebral infarction (Figure 3).

\section{The Neurological Deficit Score}

Thirty trials with 3518 participants measured the neurological deficit score, and there were two evaluation criterions, including 


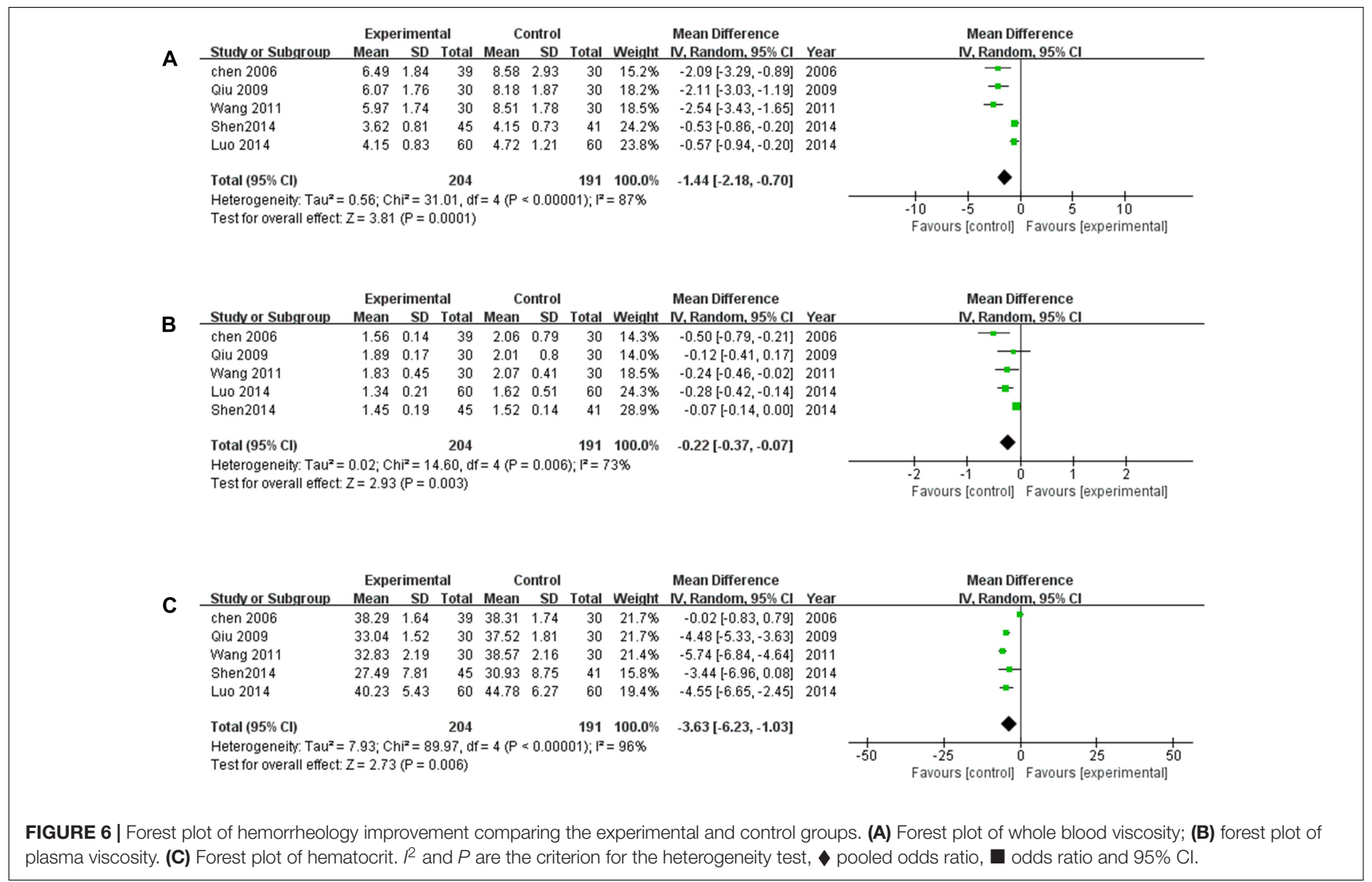

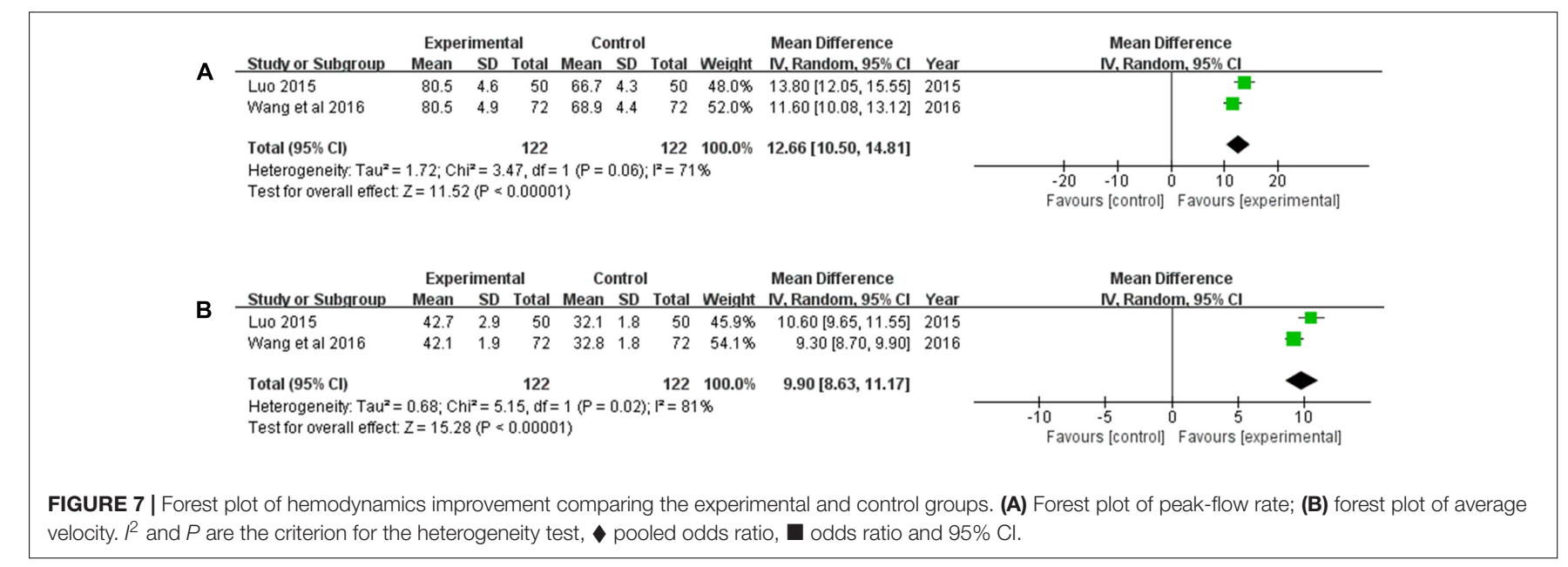

the National Institutes of Health Stroke Scale (NIHSS) (Chen and Wang, 2009; Wu, 2009; Chen, 2011; Wang et al., 2011; Wang, 2011, 2012; Jia et al., 2014; Wei and Shi, 2014; Yin et al., 2014; An et al., 2015; Lu et al., 2016; Wang and Wang, 2016), and the Chinese Stroke Scale (CSS, the fourth national cerebral vascular disease in 1995) (Zhang et al., 2006; Lian and Dong, 2010; Han et al., 2011; Li and Hou, 2011; Qin et al., 2011; Tong and Zhu, 2011; Li et al., 2012; Li and Su, 2012; Tong and Zhu, 2012; Dong and Fu, 2013; Lou, 2013; Qian and Jia, 2013; Luo, 2014; Wang et al., 2014; Zhang, 2014; Wang and
Huang, 2015; Zhao et al., 2015; Lin, 2016) used. As shown in Figure 4, there were substantial heterogeneity both in the two subgroups $\left(P<0.00001, I^{2}=92 \% ; P<0.00001, I^{2}=87 \%\right)$. A random effects model was used to pool this meta-analysis. The result of this meta-analysis showed that the neurological deficit score of patients in the experimental group was much lower, compared with the control group according to both of the two evaluation criterions $(M D=-3.44,95 \%$ CI $[-4.52$, $-2.36], P<0.00001 ; M D=-5.72,95 \%$ CI $[-6.94,-4.50]$, $P<0.00001)$. 

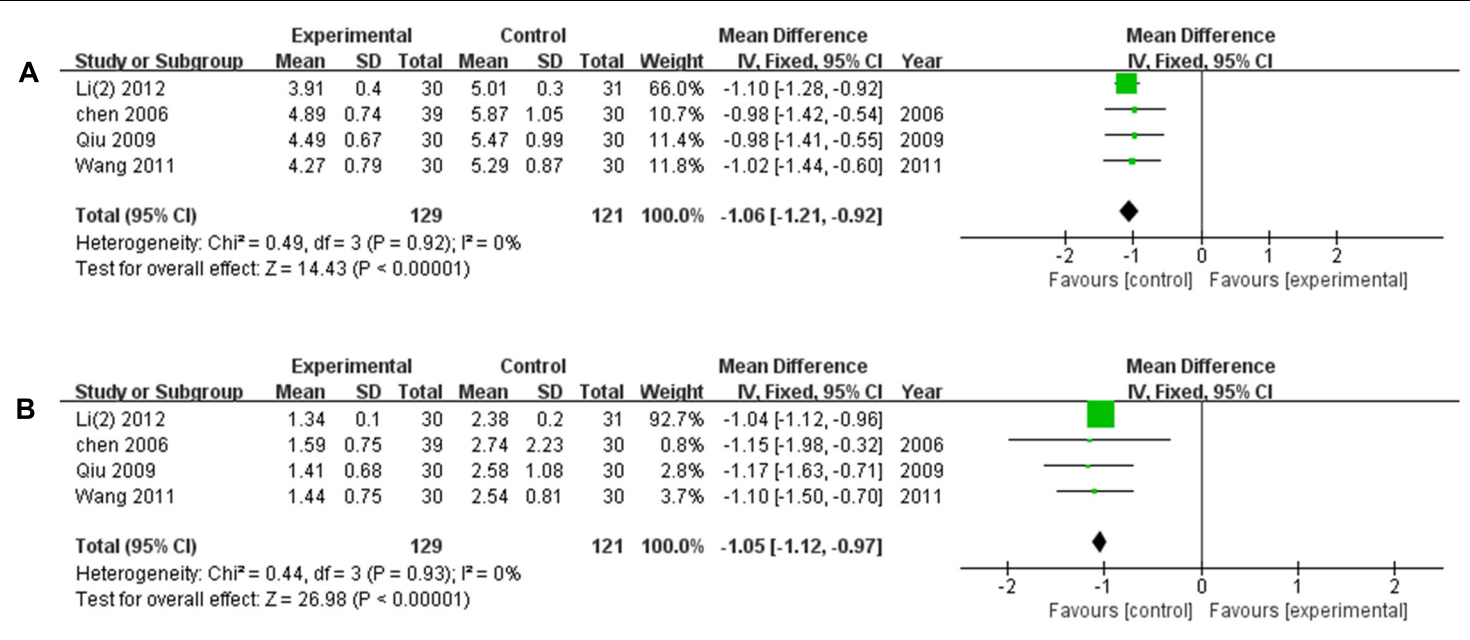

FIGURE 8 | Forest plot of blood lipid amelioration comparing the experimental and control groups. (A) Forest plot of cholesterol; (B) forest plot of triglyceride. I $^{2}$ and $P$ are the criterion for the heterogeneity test, $\bullet$ pooled odds ratio, $\mathbf{\square}$ odds ratio and $95 \% \mathrm{Cl}$.

\section{Serum Levels of MMPs}

The serum levels of MMP-2 and MMP-9 were measured. There were two studies (Sun, 2014; Cai and Zhao, 2016) involving 192 patients measured the level of MMP-2, and 5 studies with 504 participants measured the level of MMP-9. The randomeffects model was used to pool this meta-analysis for the significant heterogeneity of them $\left(P=0.04, I^{2}=76 \%\right.$; $P=0.02$, $\left.I^{2}=66 \%\right)$. The pooled analysis showed that compared with the $\mathrm{CT}$, the XNJ could significantly reduce the serum levels of MMP-2, and MMP-9 (MD $=-11.24,95 \%$ CI $[-20.83,-1.65]$, $P=0.02 ; M D=-25.08,95 \%$ CI $[-35.49,-14.67], P<0.00001)$ (Figure 5).

\section{Hemorrheology Improvement}

The hemorrheology, including whole blood viscosity (WBV), plasma viscosity (PV), and hematocrit (HCT) was measured in five studies (Chen, 2006; Qiu, 2009; Wang et al., 2011; Luo, 2014; Shen et al., 2014). The random-effects model was used for the substantial heterogeneity of the three subgroups $(P<0.00001$, $\left.I^{2}=87 \% ; P=0.006, I^{2}=73 \% ; P<0.00001, I^{2}=96 \%\right)$. As shown in Figure 6, XNJ was more effective in improving hemorrheology by reducing WBV, PV, and HCT comparing with $\mathrm{CT}(M D=-1.44,95 \% \mathrm{CI}[-2.18,0.70], P=0.001 ; M D=-0.22$, 95\% CI $[-0.37,-0.07], P=0.003 ; M D=-3.63,95 \%$ CI $[-6.23$, $-1.03], P=0.006)$.

\section{Hemodynamics Improvement}

There were two studies (Luo et al., 2015; Wang and Jiang, 2016) reporting the hemodynamics (peak-flow rate, and average velocity) improvement. Heterogeneity between the two studies was significant $\left(P=0.06, I^{2}=71 \% ; P=0.02, I^{2}=81 \%\right)$. Hence, a random-effects model was used to pool the meta-analysis. The results revealed that compared with the CT, XNJ could remarkably increase the peak-flow rate, and average velocity $(M D=12.66,95 \%$ CI $[10.50,14.81], P<0.00001 ; M D=9.90$, 95\% CI [8.63, 11.17], $P<0.00001)$ (Figure 7).

\section{Blood Lipid Amelioration}

There were four studies (Chen, 2006; Qiu, 2009; Wang et al., 2011; Li and $\mathrm{Su}, 2012$ ) reported the amelioration of blood lipid after the treatment of XNJ and CT, respectively. There was no significant heterogeneity among the individual trails $\left(P=0.92, I^{2}=0 \% ; P=0.93, I^{2}=0 \%\right)$. The meta-analysis, using the fixed-effects model, revealed that compared with the $\mathrm{CT}$, XNJ could significantly reduce the levels of cholesterol and triglyceride in blood $(M D=-1.06,95 \%$ CI $[-1.21,-0.92]$, $P<0.00001 ; M D=-1.05,95 \%$ CI $[-1.12,-0.97], P<0.00001)$ (Figure 8).

\section{Clinical Symptoms Improvement}

The clinical symptoms, including ADL, GCS, and infarct size were measured. There were five trails (Wu et al., 1999; Wang et al., 2011; Luo, 2014; Lu et al., 2016; Lu and Liu, 2016) reported the ADL improvement. As shown in Figure 9A, XNJ could significantly improve the ADL of patients with cerebral infarction, compared with the CT $(M D=10.23,95 \%$ CI [9.47, 10.99], $P<0.00001)$. There was no significant heterogeneity among the individual studies $\left(P=0.67, I^{2}=0 \%\right)$, and a fixed-effects model was used to pool this meta-analysis. As for coma scale improvement, there was no significant difference between the experimental and control groups $(M D=1.00,95 \%$ CI $[-0.96,2.96]$, $P=0.32$ ) (Figure 9B). Additionally, XNJ could significantly decrease the infarct size $(M D=-1.83,95 \%$ CI $[-2.49,-1.16]$, $P<0.00001)$. Heterogeneity between the studies was substantial, and a random-effects model was used $\left(P=0.03, I^{2}=78 \%\right)$ (Figure 9C).

\section{Publication Bias}

A funnel plot was used to evaluate the publication bias. A total of 38 trails included in the funnel plot of the overall response rate. As shown in Figure 10, there was no significant asymmetry observed. 


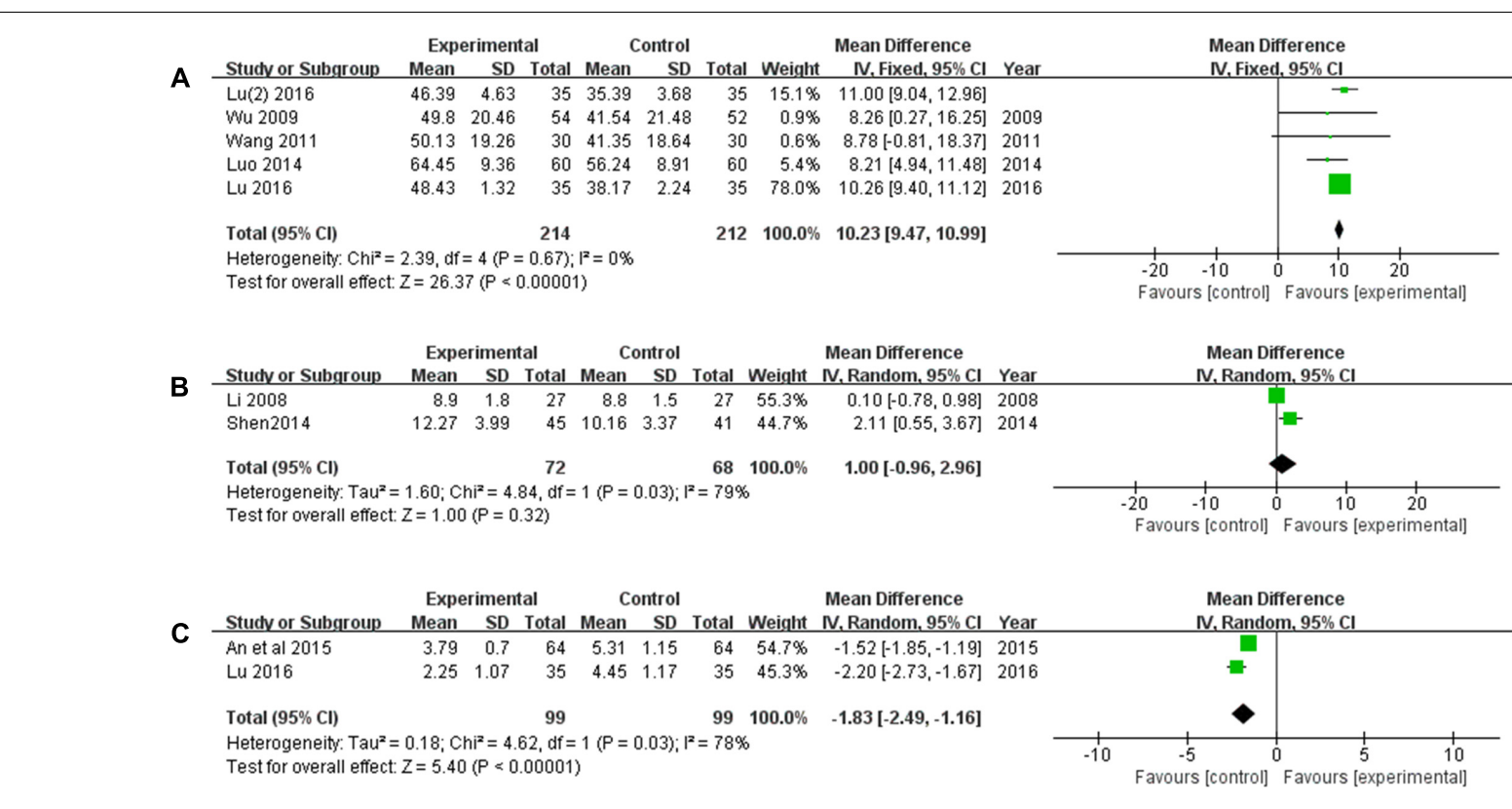

FIGURE 9 | Forest plot of clinical symptom improvement comparing the experimental and control groups. (A) Forest plot of activities of daily living; (B) forest plot of Glasgow Coma Scale; (C) forest plot of infarct size. $l^{2}$ and $P$ are the criterion for the heterogeneity test,

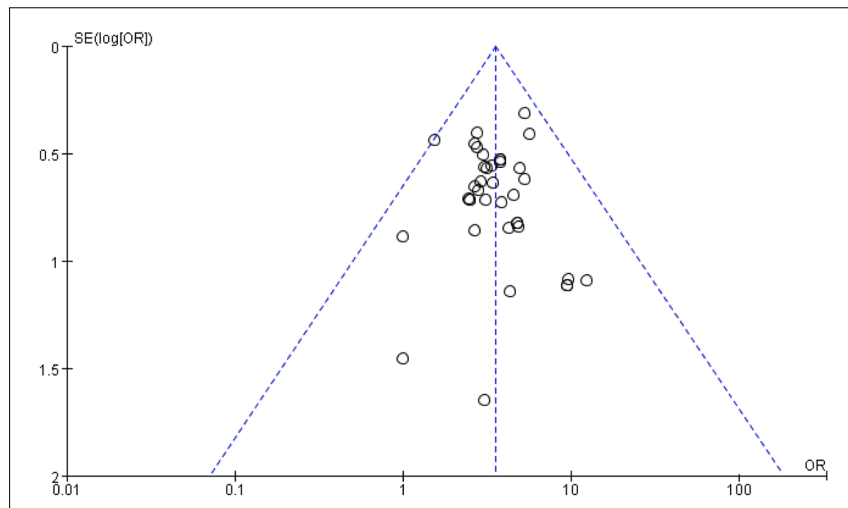

FIGURE $\mathbf{1 0}$ | Funnel plot for the publication bias of the overall response rate.

\section{DISCUSSION}

Cerebral infarction, known as ischemia stroke, is one of the common cerebrovascular diseases with high morbidity and mortality and seriously endangering the health and daily life of patients (Broussalis et al., 2011). It has been widely recognized one of the primary causes of death and disability both in developed and developing countries (Broussalis et al., 2011). CT, including thrombolysis, improving microcirculation, the applying of neuroprotective agents restoring, blood supply to ischemic area, controlling cerebral edema, preventing and treating complications, controlling hypertension, reducing blood viscosity, etc. is the main clinical therapy for the patients with cerebral infarction in current years. However, the motor weakness on one or both sides of body may be caused by the frequently used of conventional agents (Li et al., 2014). Therefore, the more effective agents for cerebral infarction patients are desirable.

Traditional Chinese medicine has been used to treat stroke in China during the past 2,000 years. Advanced pharmaceutical technologies have led to the development of many oral agents and injections for the prevention and treatment of stroke that are based on well-known traditional Chinese medical prescriptions (Wu et al., 2007). It has been demonstrated that XNJ to be an effective agent for reducing brain injuries, and enhancing functional recovery. However, a comprehensive and systematic evaluation of XNJ for the treatment of cerebral infarction is rare, according to current rigorous international standards ( $\mathrm{He}$ et al., 2012). The aim of this review was to provide an internationally accessible systematic review of the clinical efficacy and safety of $\mathrm{XNJ}$ for the cerebral infarction.

A recent meta-analysis indicated that XNJ could significantly increase efficacy rate, decrease neurological deficit scores and the serum level of TNF- $\alpha$ of the patients of stroke. The statistical analysis found that the primary mechanism of XNJ activity was neuroprotective effect via improvement of cerebral circulation and blood flow and a reduction of cerebral edema, ferritin, and inflammation (Peng et al., 2014). In this review, we further explored the effect of XNJ on cerebral infarction and provided extended findings. First of all, overall response rate and neurological deficit score were chosen to measure the clinical efficacy of XNJ according to the "the criteria for the degree of defect of the clinical function of the patients in stroke" in the fourth academic conference on cerebrovascular disease (The Fourth National Academic Conference on Cerebrovascular Diseases, 1996), which were direct correlated to improvement of patients with cerebral infarction. Compared 
with CT alone, XNJ combined with CT was associated with relatively higher overall response rate and lower neurological deficit score $(P<0.00001, P<0.00001$, respectively). The combination therapy also alleviated the clinical symptom of patients with cerebral infarction, including improving ADL, reducing infarction size $(P<0.00001$, $P<0.00001$, respectively). It did not result in a statistically significant improvement of the GCS $(P=0.32)$. The result of GCS improvement was based on two small-samples. Therefore, additional trails are necessary for a better data basis.

Meanwhile, the mechanism of XNJ activity for cerebral infarction was explored. The accumulating data revealed that MMPs are deleterious in stroke, in particular MMP-2 and MMP-9 (Rosenberg, 2002; Fatar et al., 2005). The result of our meta-analysis suggested that based on the CT, XNJ could significantly lower the level of MMP-2 and MMP-9 $(P=0.02$, $P<0.00001$ ). Additionally, the hermorrheology (including WBV, $\mathrm{PV}$, and HCT) and hemodynamics ( $\mathrm{Vp}$, and $\mathrm{Vm}$ ) were measured to evaluate the treatment effect of $\mathrm{XNJ}$ on cerebral infarction. As shown in Figure 6, XNJ could significantly improve the hemorrheology by reducing WBV, PV, and HCT $(P=0.0001$, $P=0.003, P=0.006$, respectively) based on the CT. And at the same time, the hemodynamics was also significantly improved $(P<0.00001, P<0.00001)$. There were significant correlation between occurrence of ischemic stroke and dyslipidemia. When the total cholesterol (Chol) increased by $1 \mathrm{mmol} / \mathrm{L}$, the incidence of stroke will increase by 25\% (Zhang et al., 2003; Huang and Zhang, 2011). In the systematic review, four trails mentioned the blood lipid change, including Chol and TG. The meta-analysis demonstrated that there were significant differences between the experimental and control groups in Chol and TG $(M D=-1.06$, 95\% CI $[-1.21,-0.92], P<0.00001 ; M D=-1.05,95 \% \mathrm{CI}$ $[-1.12,-0.97], P<0.00001)$. Accordingly, blood lipids level may be proposed as one of the important indicators for the evaluation of efficacy of XNJ for treating cerebral infarction in the further clinical studies.

The efficacy and adverse events associated with XNJ in treatment of cerebral infarction were explored via extensive

\section{REFERENCES}

An, N., Xie, J. D., Xu, Y., and Yang, X. F. (2015). Efficacy of Xingnaojing injection for cerebral infarction in elderly patients with and its' impact on serum IL-18. Eval. Anal. Drug Use Hosp. China 6, 735-737.

Broussalis, E., Killer, M., McCoy, M., Harrer, A., Trinka, E., and Kraus, J. (2011). Current therapies in ischemic stroke. Part A. Recent developments in acute stroke treatment and in stroke prevention. Drug Discov. Today 17, 296-309. doi: 10.1016/j.drudis.2011.11.005

Cai, L. L., and Zhao, L. J. (2016). Clinical effect of Xingnaojing in treating senile cerebral infarction. Chin. J. Cardiovasc. Rehabil. 5, 542-545.

Chen, B. P., and Wang, J. M. (2009). Effect of Xingnaojing on the level of serum S-100B protein in patients with acute cerebral infarction. Chin. J. Drug Appl. Monit. 3, 138-140.

Chen, F. Q. (2006). Clinical observation on Xingnaojing injection in treating acute cerebral infarction. J. Emerg. Tradit. Chin. Med. 15, 863-865.

Chen, J. N. (2011). Clinical observation of Xingnaojing injection in treating 30 cases of acute cerebral infarction. China Mod. Med. 7, 76-79. researches and strict methodologies. However, there are limitations to this research, such as the quality of the included data for the original trials. Many RCTs do not employ strict methodologies. Additionally, most of the involving patients were Chinese. It is necessary to including more varied population sample. The sample size, selection criteria varied for the included studies. We were unable to perform a subgroup analysis.

\section{CONCLUSION}

These findings reflect that XNJ may significantly improve overall response rate, neural functional, Hemodynamics and ADL, and decrease the blood viscosity, serum levels of MMPs, the level of blood lipid and infarction size. It is likely that XNJ also decreases the Glasgow Coma Scale, but the results were not statistically significant. However, our findings must be interpreted with caution because of the small sample size and limitations of the study. Several rigorous, large-scale RCTs are necessary to confirm these results.

\section{AUTHOR CONTRIBUTIONS}

$\mathrm{XM}, \mathrm{YY}$, and TW performed the search and wrote the manuscript. NC and QX analyzed the data. YY, TW, and XH performed the data extraction. XM and JW designed the study and amended the paper.

\section{FUNDING}

The authors wish to thank reviewers for their critical comments provided during revision and also wish to thank all authors of references. This work was financially supported by grants from National Natural Science Foundation of China (81473371, 81703725, and J1310034) and Sichuan Province Clinical Chinese Pharmacy Science and Technology Innovation Youth Team (2017TD0001).

Deng, L., Liu, X. D., Zhang, Y. B., and Li, J. M. (2011). Advances in the treatment of acute cerebral infarction. Chin. Gen. Pract. 14, 825-829.

Dong, L. H., and Fu, J. L. (2013). Clinical observation on Xingnaojing injection in treating fever after acute cerebral infarction. J. Emerg. Tradit. Chin. Med. 3, 469-470.

Fatar, M., Stroick, M., Griebe, M., and Hennerici, M. (2005). Matrix metalloproteinases in cerebrovascular diseases. Cerebrovasc. Dis. 20, 141-151. doi: $10.1159 / 000087197$

Guo, Y. (2013). Effect of Xing-naojing and Shengmai injection on PAR1 and AQP4 expressions in rats after intracerebral hemorrhage. J. Neurol. Sci. 333, e153-e154. doi: 10.1016/j.jns.2013.07.649

Guo, Y., Yan, S. H., Xu, L. P., Zhu, G., Yu, X., and Tong, X. (2014). Use of Angong Niuhuang in treating central nervous system diseases and related research. Evid. Based Complement. Alternat. Med. 34, 52-54. doi: 10.1155/2014/346918

Han, Y. Q., Liu, X., and Hu, G. Y. (2011). Clinical observation on Xingnaojing in treating large area cerebral infarction. China Pract. Med. 23, 122-123.

Hata, J., Doi, Y., Ninomiya, T., Fukuhara, M., Ikeda, F., Mukai, N., et al. (2011). Combined effects of smoking and hypercholesterolemia on the risk of stroke 
and coronary heart disease in Japanese: the Hisayama study. Cerebrovasc. Dis. 31, 477-484. doi: 10.1159/000324392

He, J., Kwon, Y., Li, C., Zhang, X. Q., and Zhao, J. G. (2012). Several considerations in using traditional Chinese patent medicine for cerebral infarction. Chin. J. Integr. Med. 18, 571-574. doi: 10.1007/s11655-012-1186-8

He, X. J., Qin, X. H., and Liu, Y. L. (2007). Borneol on anti-cerebral ischemia. Shanxi Med. J. 9, 794.

He, X. J., Qing, L. M., and Liu, Y. L. (2006). The protective effect of borneol on experimental cerebral ischemia. J. Guangdong Coll. Pharm. 2, 171.

Higgins, J. P., Thompson, S. G., Deeks, J. J., and Altman, D. G. (2003). Measuring inconsistency in meta-analyses. BMJ 327, 557-560. doi: 10.1136/bmj.327. 7414.557

Huang, J. S., and Zhang, Z. M. (2011). Clinical studies on the relations of acute cerebral infarction and lipid. China Foreign Med. Treat. 30, 45-46.

Jia, Y. J., Tong, Y., and Min, L. Q. (2014). Effect of Xingnaojing injection on serum high mobility group box- 1 protein, tumor necrosis factor- $\alpha$, interleukin18 and soluble vascular cell adhesion molecule-1 in aged patients with cerebral infarction. Chin. J. Gerontol. 9, 2395-2397.

Jiang, C. W. (2010). Effect of Xingnaojing on the clinical efficacy and recovery of neurologic deficits in patients with acute cerebral infarction. Jiangxi J. Tradit. Chin. Med. 10, 29-30.

Johnston, S. C., Mendis, S., and Mathers, C. D. (2009). Global variation in stroke burden and mortality: estimates from monitoring, surveillance, and modeling. Lancet Neurol. 8, 345-354. doi: 10.1016/S1474-4422(09)70023-7

Li, H. B., and Su, X. (2012). Effect of Xingnaojing injection on serum interleukin-6, 12 in patients with acute cerebral infarction and its clinical significance. Cap. Med. 16, 39-40.

Li, L., Zhang, H., Meng, S. Q., and Qian, H. Z. (2014). An updated meta-analysis of the efficacy and safety of acupuncture treatment for cerebral infarction. PLoS ONE 9:e114057. doi: 10.1371/journal.pone.0114057

Li, W. X., and Hou, B. J. (2011). Clinical observation on acute cerebral infarction treated by. Hubei J. Tradit. Chin. Med. 3, 32-33.

Li, X., Li, Y. H., Fang, Y., Fang, J., Li, X. H., Huang, R., et al. (2012). Observation on therapeutic effect of Xingnaojing injection on acute large area cerebral infarction. Chin. J. Pract. Nerv. Dis. 22, 76-78.

Li, Y. Y., Zhang, P., and Deng, H. B. (2008). Effect of Xingnaojing injection on neurological function in patients with acute cerebral infarction. China J. Chin. Mater. Med. 33, 2179.

Lian, Q. R., and Dong, H. L. (2010). Clinical observation on Xingnaojing injection in treating acute cerebral infarction. J. Emerg. Tradit. Chin. Med. 3, 430-431.

Liao, Y., Wu, J. F., Long, G. H., and Long, Z. W. (2015). Effect of Xingnaojing injection on serum IL-8 and IL-10 in patients with acute cerebral infarction. Jilin Med. J. 15, 3292-3293.

Lin, Y. Q. (2016). Effect of Xingnaojing injection on female patients with acute cerebral infarction. Shenzhen J. Integr. Tradit. Chin. West. Med. 16, 6-7.

Lou, Y. F. (2013). Clinical study on the effect of Xingnaojing injection on serum IL-1 and IL-6 in patients with acute cerebral infarction. J. Emerg. Tradit. Chin. Med. 5, 984-985.

Lu, Y. H., and Zhang, Y. T. (2009). Effect of Xingnaojing injection on plasma endothelin and nitric oxide in patients with acute cerebral infarction. Chin Med. J. Metallurg. Ind. 5, 589-590.

Lu, Z. G., Huang, J. B., Liu, Y., and Lv, X. Q. (2016). Effects of Xingnaojing injection on Keap1-Nrf2/ARE oxidative stress pathway in acute cerebral infarction. Guangdong Med. J. 20, 3127-3129.

Lu, Z. G., and Liu, Y. (2016). Effect of Xingnaojing injection on curative effect and related index of blood stasis syndrome in acute cerebral infarction. China Pharm. 21, 2898-2900.

Luo, J. (2014). Clinical observation of Xingnaojing injection combined with western medicine in treating acute cerebral infarction. J. Tradit. Chin. Med. Univ. Hunan 5, 52-54.

Luo, J. F., Li, W. H., Liu, Z. X., and Xie, T. (2015). Effect of Xingnaojing injection on cerebral hemodynamics in elderly patients with cerebral infarction. Strait Pharm. J. 9, 143-144.

Luo, J. N. (2010). New progress on treating acute cerebral infarction. Pract. J. Cardiac. Cereb. Pneumal. Vasc. Dis. 18, 1546-1547.

Lv, F. Y. (2014). Effect of Xingnaojing injection on the curative effect of patients with cerebral infarction and the level of MMP-2 and MMP-9. Chin. J. Pract. Nerv. Dis. 21, 64-65.
Ma, B., Liu, L., Zhang, Y., Sun, Y., Zhao, Z., High, Y., et al. (2014). Xingnaojing injection attenuates neurologic deficits against focal ischemic injury in strokeprone renovascular hypertensive rats. Chin. J. Integr. Med. Cardio Cerebrovasc. Dis. 2, 212-214.

Peng, W. J., Yang, J. J., Wang, Y., Wang, W. H., Xu, J. X., Wang, L. X., et al. (2014). Systematic review and meta-analysis of randomized controlled trials of Xingnaojing treatment for stroke. Evid. Based Complement. Alternat. Med. 2014:210851. doi: 10.1155/2014/210851

Qian, L. L., and Jia, K. (2013). Effect of Xingnaojing injection on cerebral protection and interleukin- 6 and interleukin- 8 in patients with acute cerebral infarction. Chin. Tradit. Patent Med. 8, 1633-1636.

Qin, Z. Y., Lin, C. R., and Zhang, W. Z. (2011). Effect of Xingnaojing injection on consciousness disorder in patients with cerebral infarction. Youjiang Med. 6, $740-742$.

Qiu, Y. F. (2009). Clinical experience of Xingnaojing injection in treating 30 cases of acute cerebral infarction. J. Gannan Med. Univ. 1, 102-103.

Rosenberg, G. A. (2002). Matrix metalloproteinases in neuroinflammation. Glia 39, 279-291. doi: 10.1002/glia.10108

Shen, C. Q., Liu, Z. F., Xi, Y., Wang, J., Xiang, Z. B., and Qian, Y. M. (2014), Clinical Study on Xingnaojing injection in treating acute cerebral infarction with consciousness disorder. Yunnan J. Tradit. Chin. Med. Mater. Med. 35, 8.

$\mathrm{Su}$, J. (2008). Clinical observation on treatment of 39 cases of acute cerebral infarction with Xingnaojing injection. Chin. J. Mod. Drug Appl. 2, $84-85$.

Sun, J. (2014). Effect of Xingnaojing injection on the expression of soluble tumor necrosis factor-related apoptosis-inducing ligand, matrix metalloproteinase2 and -9 in elderly patients with cerebral infarction. Chin. J. Gerontol. 9, 2376-2377.

Sun, Y. M., Wang, Y. T., and Jiang, L. X. (2015). Effect of Xingnaojing injection on serum matrix metalloproteinase - 9 in patients with cerebral infarction. Cardiovasc. Dis. J. Integr. Tradit. Chin. West. Med. 36, 42-43.

Sze, F. K., Yeung, F. F., Wong, E., and Lau, J. (2005). Does Danshen improve disability after acute ischaemic stroke? Acta Neurol. Scand. 11, 118-125.

The Fourth National Academic Conference on Cerebro-vascular Diseases (1996). Scores criteria for clinical neurological function deficiency of stroke patients. J. Chin. J. Neurol. 29, 381-383.

Tong, X. W., and Zhu, J. (2011). Observation on therapeutic effect of Xingnaojing injection in treating senile acute cerebral infarction. Chin. J. Integr. Med. Cardio Cerebrovasc. Dis. 8, 943-944.

Tong, X. W., and Zhu, J. (2012). Observation on therapeutic effect of Xingnaojing injection in treating acute severe cerebral infarction. Mod. J. Integr. Tradit. Chin. West. Med. 4, 387-388.

Wang, B., Wang, X. H., Yao, X. Y., Xu, X., Jia, H. J., and Yang, L. L. (2014). Clinical observation of Xingnaojing injection combined with conventional therapy in treating acute cerebral infarction with consciousness disturbance. China Pract. Med. 27, 133-134.

Wang, F. (2011). Clinical effect of Xingnaojing injection in treating cerebral infarction and its influence on cytokines. Mod. J. Integr. Tradit. Chin. West. Med. 34, 4362-4363.

Wang, H., and Huang, D. (2015). Therapeutic effect of Xingnaojing injection on neurologic deficit after cerebral infarction. Chin. J. Pract. Nerv. Dis. 6, 126-127.

Wang, M. L. (2012). Effect of Xingnaojing injection on neurological deficit and serum IL-12 expression in elderly patients with cerebral infarction. Chin. J. Gerontol. 32, 5389-5390.

Wang, S., and Wang, D. Y. (2016). Curative effect of Xingnaojing injection on cerebral hemodynamics in elderly patients with cerebral infarction. J. Clin. Med. 10, 1975-1976.

Wang, Y., and Jiang, Y. T. (2016). Observation on therapeutic effect of Xingnaojing injection in treating senile cerebral infarction. China Pract. Med. 17, 156-157.

Wang, Y. Q., Zhang, W. K., Kang, G. C., and Zhang, J. S. (2011). Clinical observation on 40 cases of acute cerebral infarction treated by Xingnaojing injection. Chin. J. Basic Med. Tradit. Chin. Med. 17, 4.

Wei, X. N., and Shi, Y. H. (2014). Xing Nao Jing injection in treating 50 cases of acute cerebral infarction. West China J. Tradit. Chin. Med. 10, 88-89.

Wu, B., Liu, M., Liu, H., Li, W., Tan, S., Zhang, S., et al. (2007). Meta-analysis of traditional Chinese patent medicine for ischemic stroke. Stroke 38, 1973-1979. doi: 10.1161/STROKEAHA.106.473165 
Wu, D. F. (2008). Effect of Xingnaojing injection on malondialdehyde in patients with acute cerebral infarction and its clinical efficacy. Public Med. Forum Mag. 25, 796-797.

Wu, Q. C. (2009). Effect of Xingnaojing injection in treating acute cerebral infarction and intervening effect of inflammatory. J. Emerg. Tradit. Chin. Med. 11, 1809-1810.

Wu, Y. S., Wang, Z., and Jiang, L. P. (1999). Effect of Xingnaojing injection on expression of $\mathrm{CD}+(54)$ in monocytes of patients with acute cerebral infarction. Pract. J. Integr. Tradit. Chin. West. Med. 6, 33-34.

Xiong, W. Z., Chen, S., and Li, J. (2016). Observation on therapeutic effect of Xingnaojing injection in treating acute cerebral infarction. Guide China Med. $28,22-23$.

Yang, B. (2011). Effect of Xingnaojing injection on neurological deficit and serum IL-12 expression in elderly patients with cerebral infarction. Chin. J. Gerontol. 32, 340-399.

Yang, N. (2016). Effect of Xingnaojing injection on cerebral hemodynamics in patients with cerebral infarction and its clinical efficacy. J. Liaoning Med. Univ. $1,37-39$.

Yin, P., and Wei, Y. P. (2014). Effect of Xingnaojing injection on serum matrix metalloproteinase-9. Med. Recapitulate 13, 2430-2431.

Yin, S. L., Po, S. F., and Liu, Y. (2014). Clinical observation on Xingnaojing in treating acute cerebral infarction with consciousness disorder. Chin. J. Pract. Nerv. Dis. $17,6$.

Zang, Z. L., Wang, S. J., and Cao, H. Q. (2011). Clinical observation of Xingnaojing injection in treating coma of cerebral infarction. Mod. J. Integr. Tradit. Chin. West. Med. 11, 1323-1324.
Zeng, G. J. (2013). Clinical observation on Xingnaojing injection in treating cerebral infarction. Chin. Med. Innov. 26, 48-49.

Zhang, M. Q., Wang, D., and Xu, J. (2006). Clinical observation on 60 cases of acute severe cerebral infarction treated. Jilin J. Tradit. Chin. Med. 9, 11.

Zhang, X., Patel, A., Horibe, H., Wu, Z., Barzi, F., Rodgers, A., et al. (2003). Cholesterol, coronary heart disease, and stroke in the Asia Pacific region. Int. J. Epidemiol. 32, 563-572. doi: 10.1093/ije/dyg106

Zhang, X. L. (2014). Application of Xingnaojing injection in the treatment of neurologic deficits after cerebral infarction. Womens Health Res. 8, 194-196.

Zhao, L. R., Piao, C. S., Murikinati, S. R., and Gonzalez-Toledo, M. E. (2015). The role of stem cell factor and granulocyte-colony stimulating factor in treatment of stroke. Recent Pat. CNS Drug Discov. 8, 2-12. doi: 10.2174/ 1574889811308010002

Zhou, K. (2010). Progress in the treatment of acute cerebral infarction. Pract. J. Car Cereb. Pneumal. Vasc. Dis. 18, 1726-1728.

Conflict of Interest Statement: The authors declare that the research was conducted in the absence of any commercial or financial relationships that could be construed as a potential conflict of interest.

Copyright (C) 2017 Ma, Yang, Chen, Xie, Wang, He and Wang. This is an open-access article distributed under the terms of the Creative Commons Attribution License (CC BY). The use, distribution or reproduction in other forums is permitted, provided the original author(s) or licensor are credited and that the original publication in this journal is cited, in accordance with accepted academic practice. No use, distribution or reproduction is permitted which does not comply with these terms. 